This is a postprint version of the following published document:

C. Vázquez and D. S. Montero."Temperature impairment characterization in radio-over-multimode fiber systems", Proc. SPIE 8282, Broadband Access Communication Technologies VI, 82820H (January 24, 2012); doi:10.1117/12.919702

(C) 2012 Society of Photo Optical Instrumentation Engineers. One print or electronic copy may be made for personal use only. Systematic electronic or print reproduction and distribution, duplication of any material in this paper for a fee or for commercial purposes, or modification of the content of the paper are prohibited. 


\title{
Temperature impairment characterization in Radio-over-Multimode Fiber systems
}

\author{
C. Vázquez ${ }^{* a}$, D.S. Montero ${ }^{\mathrm{a}}$ \\ ${ }^{a}$ Electronics Technology Dpt., Universidad Carlos III de Madrid, Avda. de la Universidad 30, 28911, \\ Leganés (Madrid), Spain
}

\begin{abstract}
An emerging theme in next-generation access research includes seamless wireline-wireless convergence addressed by Radio-over-Fiber (RoF) technologies. Optical cabling solutions offer the possibility for semi-transparent transport through the access network microwave to mm-wave radio carriers commonly employed for creating high-capacity picocell wireless networks, attending present demands from the wireless technologies, with portable/mobile devices converging with photonics. Advanced RoF techniques can efficiently generate and transport such carriers, and deliver them to simplified antenna stations or radio access points (RAPs). Thus, they can convey high data rates in comprehensive modulation formats on multiple-GHz carriers in MMF networks.

Selective mode-launching schemes combined with the use of narrow linewidth optical sources are experimentally demonstrated to enable broadband RF, microwave and mm-wave transmission in short- and middle-reach distances over silica-based multimode optical fibers (MMFs); and are reviewed in this paper. However, arbitrary operating conditions, such as the temperature dependence in the fiber link, impose a great challenge for the extension of the RoMMF technology. Temperature impairment characterization is analyzed over the broadband transmission bands that are present, under certain operating link conditions, in the frequency response performance of MMF to support multiple GHz carrier delivering schemes, thus contributing to fault link prevention.
\end{abstract}

Keywords: multimode fiber (MMF), temperature dependence, Radio-over-Fiber (RoF), frequency response

\section{INTRODUCTION}

Actual trends of increasing speed and coverage in the next-generation access (NGA) services marketplace indicate further deployment of higher-capacity communications technologies well into the foreseeable future, even inside the customer's premises, with the need for short- and middle-reach links supporting data rates of Gbps and 10Gbps. It is also a matter of fact that recent interests are focused on high-quality display technologies such as high-definition (HD), threedimensional visual information (3D) or remote "face-to-face communication", which forecast requirements for data transmission speed more than $40 \mathrm{Gbps}$ by 2020 , that can be achievable only with optical networks ${ }^{[1]}$.

In addition to these bandwidth requirements, in the final link to the end users, telecommunication networks have to deliver a large variety of services with widely ranging signal characteristics. Moreover, a cost-effective and future-proof solution for bringing high bandwidth services to the individual users on a mass basis has not yet emerged and several competing technologies are under consideration: unshielded twisted copper pair (UTP), coax cable, wireless links, and optical fibers. Optical communication systems based on optical fibers allow communication signals to be transmitted not only over long distances with low attenuation but also at extremely high data rates, or bandwidth capacity. Focusing these optical fiber solutions, silica-based MMFs provide the necessary bandwidth for short- and middle-reach links at a much lower installation and component cost, compared to their SMF counterparts, due to their larger core dimensions. In addition to this, it is worth mentioning that the majority of the installed fiber cable, five out of six, corresponds to multimode fiber as recommended by the ISO/IEC 802.11. So, there is recently an important drive to utilize this existing infrastructure to support the ever increasing demand on high-speed services.

Another important point in access networks communications is within the field of the wireless signal transmission (for both mobile and data communication), namely Wireless Local Area Networks (WLANs). Wireless technologies are developing fast but there is a need to link base stations/servers to the antenna by using fixed links together with the

*cvazquez@ing.uc3m.es; phone +34 91624 9191; fax +34 916249430 
future exploitation of capacities well beyond present day standards (IEEE802.11a/b/g), which offer up to 54Mbps and operate at $2.4 \mathrm{GHz}$ and $5 \mathrm{GHz}$, as well as $3 \mathrm{G}$ mobile networks such as IMT2000/UMTS (International Mobile Telecommnications-2000/Universal Mobile telecommunications System) which offer up to 2Mbps and operate around 2GHz. Moreover, IEEE802.16, otherwise known as WiMAX, is another recent standard aiming to bridge the last mile through mobile and fixed wireless access to the end user at frequencies between $2-11 \mathrm{GHz}$. In addition, WiMAX also aims to provide Fixed Wireless Access at bit-rate in the excess of $100 \mathrm{Mbps}$ and at higher frequencies between 10$66 \mathrm{GHz}$. All these services use signals at the radio-frequency (RF) level that are analogue in nature, at least in the sense that they cannot be carried directly by digital baseband modulation. Optical cabling solutions can also offer the possibility for semi-transparent transport of these signals by using Radio-over-Fiber (RoF) technology ${ }^{[2]}$. This technology has been proposed as a solution for reducing overall system complexity by transferring complicated RF modem and signal processing functions from radio access points (RAPs) to a centralised control station (CS), thereby reducing system-wide installation and maintenance costs. Furthermore, Radio-over-Fiber (RoF) in combination with multimode fibers can be deployed within homes and office buildings for baseband digital data transmission supporting 3.5 GHz wireless signals, within the Ultra Wide Band (UWB). The UWB transmission is an emerging technology for wireless high-speed due to the high data-rate capabilities, low self-interference, low interception probability and tolerance to multi-path fading.

However, in general, low carrier frequencies offer low bandwidth and the $6 \mathrm{GHz}$ UWB unlicensed low band is not available worldwide due to coexistence concerns ${ }^{[3]}$. These include radio and TV broadcasts, and systems for (vital) communication services such as airports, police and fire, amateur radio users and many others. In contrast, the $60 \mathrm{GHz}-$ band, within millimetre-wave range, offers much greater opportunities as the resulting high radio propagation losses lead to numerous picocell sites and thus to numerous radio access points due to the limited cell coverage. These picocells are a natural way to increase capacity (i.e. to accommodate more users) and to enable better frequency spectrum utilisation. Therefore, for broadband wireless communication systems to offer the needed high capacity, it appears inevitable to increase the carrier frequencies to the range of millimetre-wave and to reduce cell sizes ${ }^{[4]}$.

In addition to the inherent bandwidth limitation of MMFs, the frequency response of MMF links depends, in general, on launching conditions, source type, variable link lengths, installation bends, connector offsets, environmental temperature changes, or the introduction of any other component along the link, which makes the MMF frequency response unpredictable under arbitrary operating conditions, which imposes a great challenge for the extension of this bandwidthdependent RoMMF performance. The potential MMF capacity for communication needs a greater exploitation to meet user requirements for higher data rates and to support the emerging multimedia applications, even more with the inclusion of wireless signals over MMF. To enable the design and utilization of MMFs with such enhanced speeds and signal characteristics, the development of an accurate frequency model is of prime importance. From most of the published models, a method relying on the propagation electric field signals has emerged ${ }^{[5,6]}$, which takes into account most of the aforementioned factors that affect the MMF frequency response. From these works the use of selective-mode launching schemes combined with the use of narrow linewidth sources to enable broadband RF, microwave and millimeter-wave in short- and middle-reach distances over optical fiber is demonstrated.

In this paper, theoretical simulations and experimental measurements showing the conditions and capability of developing MMF broadband access networks are revisited. Moreover, temperature impairment characterization is analyzed over the broadband transmission bands that are present, under certain operating link conditions, in the frequency response performance of MMF to support multiple $\mathrm{GHz}$ carrier delivering schemes, thus contributing to fault link prevention.

\section{BROADBAND TRANSMISSION IN SILICA-BASED MULTIMODE OPTICAL FIBERS}

Novel techniques to expand the MMF capabilities are continuously reported demonstrating that the frequency response of MMF does not diminish monotonically to zero after the baseband bandwidth, but tends to have repeated passbands beyond that ${ }^{[7]}$. In recent times, these high-order passbands (or secondary lobes in the frequency spectrum) and flat regions have been used in research to transmit independent streams of data (digital or analogue) complementary to the baseband bandwidth in order to exceed the aggregated transmission capacity of $\mathrm{MMF}^{[8]}$, as well as to transport microwave and millimetre-wave radio carriers, commonly employed for creating high-capacity picocell wireless networks in RoF systems, as in ${ }^{[9]}$. 


\subsection{Multimode Optical Fiber Transfer Function}

In this section a closed-form analytic expression to compute the baseband and RF transfer function of a MMF link (with fiber length $z$ ) based on the electric field propagation method is briefly presented. For a deeper comprehension works reported in ${ }^{[5,10]}$ are recommended.

From the mathematical framework it is possible to obtain the overall RF transfer function, given by Eq. (1), which provides a description of the main factors that affect the frequency response of a MMF link, which incorporates the source chirp $\alpha_{C}$ and in which $\Omega$ represents the frequency of the RF modulating signal.

$$
H(\Omega)=\sqrt{1+\alpha_{C}^{2}} \cdot e^{-\frac{1}{2}\left(\frac{\beta_{o}^{2} \Omega z}{\sigma_{c}}\right)^{2}} \cdot \cos \left(\frac{\beta_{o}^{2} \Omega^{2} z}{2}+\arctan \left(\alpha_{C}\right)\right) \cdot \sum_{m=1}^{M} 2 m\left(C_{m m} \chi_{m m}+G_{m m}\right) e^{-2 \alpha_{m} z} e^{-j \Omega \tau_{m} z}
$$

From Eq. (1) the RF frequency response of a MMF link can be divided as the product of three terms of factors. From the left to the right, the first term is a low-pass frequency response that depends on the first-order chromatic dispersion parameter $\beta_{o}^{2}$, which is assumed to be equal for all the modes guided by the fiber, and $\sigma_{c}$, which is the source coherence time directly related to the source linewidth. The second term is related to the carrier suppression effect due to the phase offset between the upper and lower modulation sidebands. Finally, the third term represents a microwave photonic transversal filtering effect in which each sample corresponds to a different mode group $m$ carried by the fiber and in which coefficients $C_{m m}, \chi_{m m}$ and $G_{m m}$ stand for the light injection efficiency, mode spatial profile impinging the detector area, and mode coupling coefficient, respectively. This last term involves that the periodic frequency response of transversal filters could permit a transmission capacity increase in such links, and results show that MMFs offer the potential for broadband transmission far from baseband through the presence of high-order resonances as well as flat regions in the frequency response in the microwave and millimetre-wave. Parameters $\alpha_{m}$ and $\tau_{m}$ represent the differential mode attenuation (DMA) effect, which causes the attenuation coefficient to vary from mode to mode, and the delay time of the guided modes per unit length, respectively.

\subsection{Silica-based MMF transfer function simulations}

Theoretical simulations have been studied with regards to different parameters affecting the frequency response, in order to determine the optimal conditions for a higher transmission bandwidth in baseband and to investigate the potentials for broadband transmission in regions far from baseband using silica-based multimode fibers. In this section some representative examples of simulations are shown.

For the simulations it has been considered a $62.5 / 125 \mu \mathrm{m}$ graded-index multimode fiber with $\mathrm{SiO}_{2}$ core doped with a $6.3 \mathrm{~mol}-\%$ of germanium $\left(\mathrm{GeO}_{2}\right)$ with intrinsic attenuation of $0.55 \mathrm{~dB} / \mathrm{km} @ 1300 \mathrm{~nm}$. Graded index exponent of the fiber has been set to a typical value of $\alpha=2.02$, analysis of the influence of slight fluctuations of this parameter can be seen in [2]. The core and cladding refractive indices have been approximated using a three-term Sellmeier function for a wavelength of $1300 \mathrm{~nm}$. The mode coupling coefficient $G_{m m}$ was defined by a Gaussian autocorrelation function with a rms deviation of $\sigma=0.0005$ and a correlation length of $\varsigma=150 \cdot a$, being $a$ the core radius. It has been assumed a free chirp source and an OverFilled Launch (OFL) condition (i.e., uniform excitation), by setting the light injection coefficient as $C_{m n}=1 / \mathrm{M}$ being $\mathrm{M}$ the total number of mode groups. DMA effects have been simulated by using the empirically function reported in $^{[11]}$, setting parameters $\rho=9$ and $\eta=7.35$. 
(a)
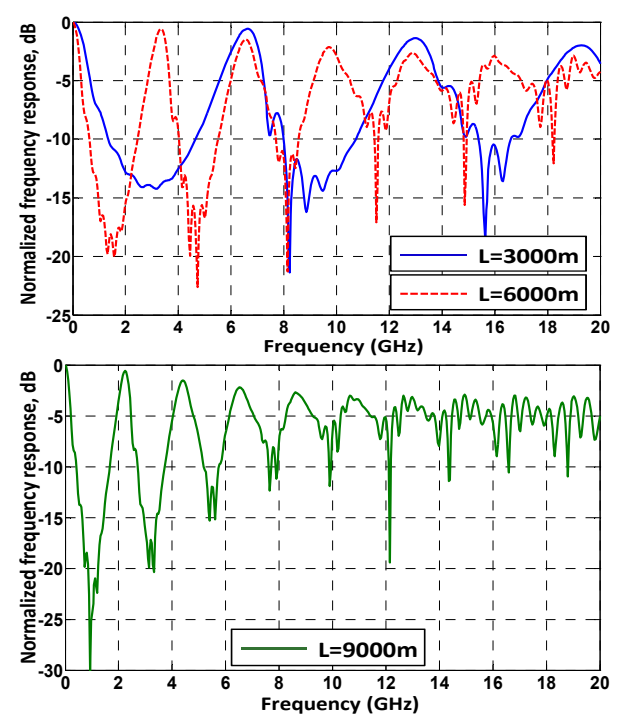

(b)

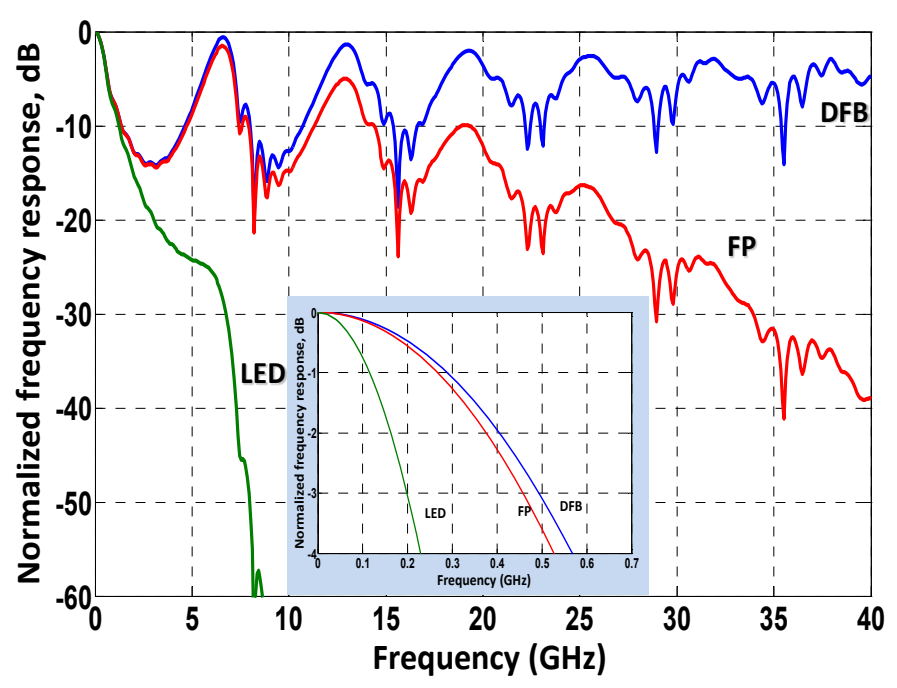

Figure 1. (a) Frequency response for different MMF link lengths. (b) Influence of the optical source temporal coherence on the frequency response for a $3 \mathrm{~km}$-long MMF link. 3-dB baseband bandwidth dependence can be seen on inset.

Fig. 1(a) shows the silica-based MMF frequency response dependence on the link length. For this figure a Distributed Feedback (DFB) laser with $50 \mathrm{MHz}$ of rms linewidth has been considered. It can be seen that higher order resonances far from baseband are slightly displaced over the frequency spectrum, being the ratio of resonance periodicities inversely proportional to the link length. On the other hand, the influence of the optical source linewidth characteristics over the frequency response as well as on the 3-dB baseband bandwidth is depicted in Fig. 1(b). Same DFB laser characteristics as Fig.1(a) have been considered. In addition, the MMF frequency responses for a multimode Fabry-Perot (FP) laser and a Light Emitting Diode (LED) with linewidths $5.5 \mathrm{~nm}$ and $30 \mathrm{~nm}$, respectively, have been included. From this figure a first resonance featuring a 3-dB bandwidth of around $1.1 \mathrm{GHz}$ in the case of the DFB and FP laser is obtained, both around a central frequency of $6.5 \mathrm{GHz}$. On the contrary, no resonance is present when a broadband light source (LED) is applied. This is due to the fact that, in this latter case, the low-pass term in Eq. (1) dominates over the other two. Furthermore, for a DFB laser source the attenuation in the higher-than-first resonances remains under similar values, even performing a flat-response region over $35 \mathrm{GHz}$. This latter performance is related to the fact that the MMF link behaves like an imperfect microwave photonic transversal filter. On the other hand, for a FP laser source the second and following resonances are rapidly decreased in optical power.

\subsection{Experimental results}

In this section, some measurement examples of the silica-based MMF transfer function are presented.

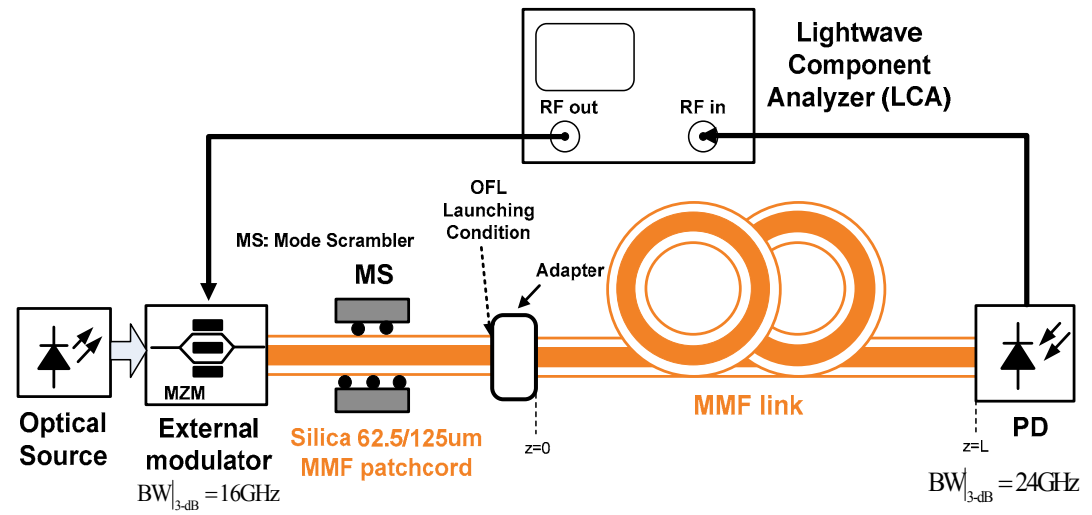

Figure 2. Block diagram of the experimental setup for the MMF link frequency response measurement, up to $20 \mathrm{GHz}$. 
The set-up schematic for the experimental measurements is shown in Fig. 2. A Lightwave Component Analyzer (LCA, Agilent $8703 \mathrm{~B}, 50 \mathrm{MHz}-20 \mathrm{GHz}$ ) has been used to measure the frequency response, using a $100 \mathrm{~Hz}$ internal filter. In all cases the laser was externally AM modulated with an RF sinusoidal signal up to $20 \mathrm{GHz}$ of modulation bandwidth, by means of an electro-optic (E/O) Mach-Zehnder modulator (model JDSU AM-130@1300nm and JDSU AM155@1550nm). The optical output of the E/O modulator was passed through a 62.5/125 $\mu \mathrm{m}$ silica-based MMF fiber patch cord plus a mode scrambler before being launched to the MMF link. This optical launching scheme provides an OFL condition for light injection. At the receiver, the frequency response is detected by using a high-speed PIN photodiode, model DSC30S, from Discovery Semiconductors. It should be mentioned that the experimental results of the silica-based MMF link shown in this section have been calibrated with regards to both the E/O intensity modulator and the photodetector electrical responses, being therefore solely attributed to the MMF fibre. It should be also noted that the ripples observed are caused by reflections in the optical system and are not features of the fiber response, although FC/APC connectors are used to minimize this effect.

$62.5 / 125 \mu \mathrm{m}$ core/cladding diameter silica-based MMF links of lengths $6 \mathrm{~km}$ and $9 \mathrm{~km}$ have been evaluated, showing their normalized frequency response up to $20 \mathrm{GHz}$ in Fig. 3, for three different types of optical sources. Focusing on Fig. 3(a), as it is expected from the theory, the response for the DFB laser (in this case operating at $1550 \mathrm{~nm}$ and with a Full Width Half Maximum -FWHM- of $100 \mathrm{kHz}$ ) behaves relatively flat at high frequencies, with maximum variations of approximately $\pm 1.5 \mathrm{~dB}$ with regards to a mean level of $3-\mathrm{dB}$ below the low frequency regime. The frequency response relative to the FP laser @1300nm with FWHM of 1.8nm, suffers from a low pass effect, for instance by a 18dB fall at $20 \mathrm{GHz}$ of a $6 \mathrm{~km}-$ long link, and no high-order resonances are observed. In the case of the Broadband Light Source (BLS) with $400 \mathrm{~nm}$ spectral range, the response falls dramatically after a few GHz.

(a)

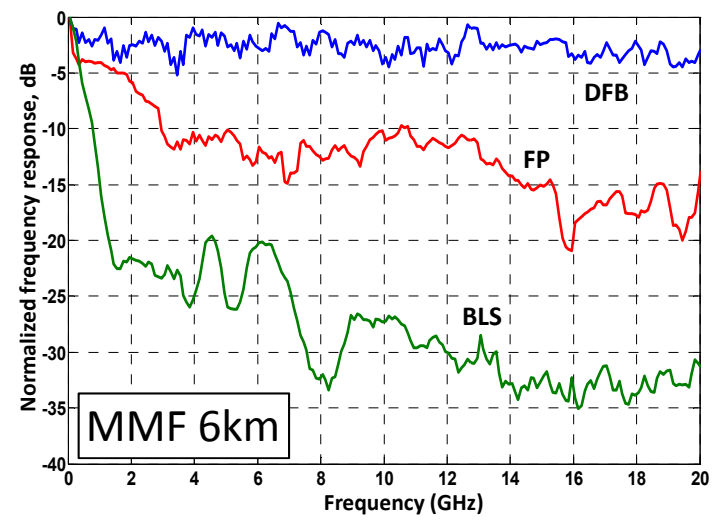

(b)

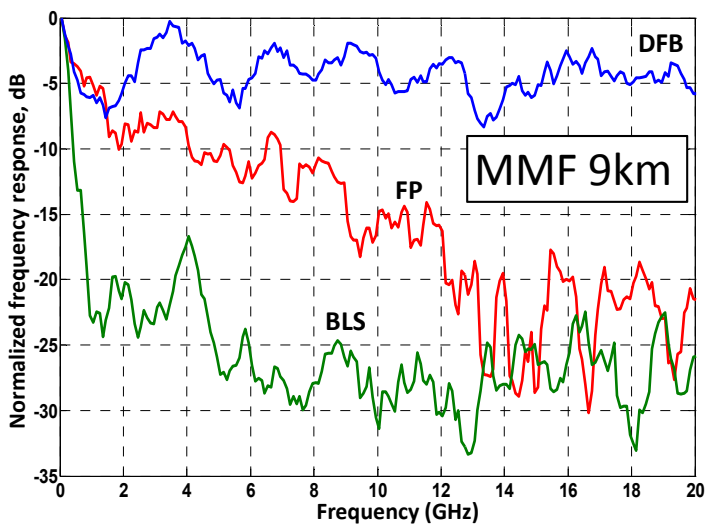

Figure 3. Measured influence of the optical source linewidth on the frequency response of (a) 3km-long link; (b) 6km-long link. DFB laser@1550nm, FP laser@1300nm, BLS: Broadband Light Source.

Evaluating the results from Fig. 3(a) and Fig. 3(b) resonances are clearly seen for the case of the DFB optical source whereas a higher attenuation is achieved for the FP case and the Broadband Light Source (BLS). Comparing these figures, it is observed that exploiting the possibility of transmitting broadband signals at high frequencies (in the microwave and mm-wave regions) is contingent on the use of narrow linewidth sources. For instance, the presence of high-order resonances and a flat region over $18 \mathrm{GHz}$ in the frequency response of the $9 \mathrm{~km}$-long MMF link for the DFB case, see Fig. 3(b), is clearly observed.

Finally, Fig. 4 shows a comparison between the frequency response predicted by the theoretical model and that of experimentally obtained showing relative good agreement between them, addressing the presence of a relatively flat region with mean level of $1.5 \mathrm{~dB}$ below the low frequency regime for the case of a $3 \mathrm{~km}$-long MMF link, see Fig. $4 \mathrm{a}$, as well as the presence of high-order resonances suitable for multiple- $\mathrm{GHz}$ carrier transmission or the case of a 9km-long MMF link. Although no accurate agreement can be expected due to the many approximations made in the theoretical analysis as well, as the amount of parameters involved in the frequency response in combination with the uncertainty of some key parameters; the results reveal a relative good agreement in the behaviour of the silica-based MMF frequency response compared to the curves predicted by the model, especially attending to the concordance in the central frequencies of the high-order resonances. 
It should be outlined that most of the experiments over the legacy MMFs were carried out applying different techniques to expand the transmission capability of the MMF link, such as selective mode-launching schemes (i.e. RML, Restricted Mode Launching) or equalization techniques at reception. However, the aim of a record data-rate transmission performance is out of the scope of this work, so, for instance, selective mode launching schemes have not been applied, but focusing on the high-order resonances performance of the MMF frequency response in terms of temperature dependence as shown in next section. Anyhow, previous frequency response measurements cover up to $20 \mathrm{GHz}$ at $1 \mathrm{Km}$ link length with $1 \mathrm{~dB}$ loss ${ }^{[12]}$, up to $50 \mathrm{GHz}$ at $500 \mathrm{~m}$ link length with $15 \mathrm{~dB}$ loss $^{[13]}$, or up to $20 \mathrm{GHz}$ at $5 \mathrm{Km}$ length with $5 \mathrm{~dB} \operatorname{loss}^{[14]}$. So these are the first measurements on Si MMF of product bandwidth lengths up to $150 \mathrm{GHz} \cdot \mathrm{Km}$, and showing MMF propagation capability beyond 3-dB bandwidth at longer link lengths.

(a)

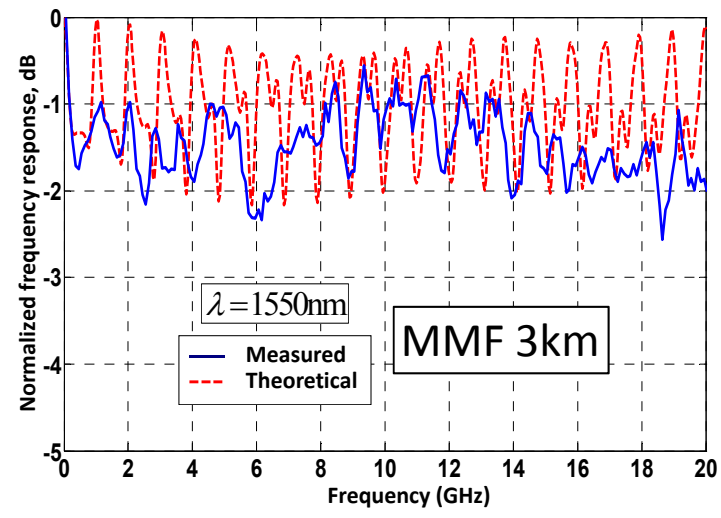

(b)

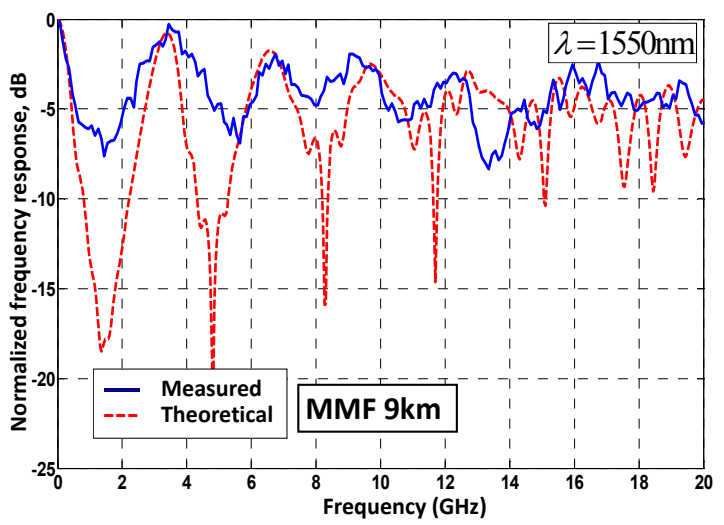

Figure 4. Theoretical and measured frequency response of 3km-long (a) and 9km-long (b) silica-based MMF link with DFB laser source at $1550 \mathrm{~nm}$.

The following section will focus on the frequency response temperature dependence in a MMF link. The location and bandwidth of the high-order resonances and the flat region is of prime importance for the deployment of a multiple-GHz carrier RoMMF system, if the latter takes advantage of the frequency response performance of silica-based MMFs.

\section{TEMPERATURE IMPAIRMENT CHARACTERIZATION}

An important aspect highlighted by the model is the great dependence of the broadband RoF transmission in the microwave and millimeter-wave regions far from baseband on the optical fiber properties. Variations in temperature have also been demonstrated to change some of the optical properties of silica-based MMF such as the graded index exponent ${ }^{[15,16]}$. This fact also imposes a great challenge for the extension of this bandwidth dependent MMF performance and, consequently, it is necessary to estimate its effect over the broadband transmission bands, for instance in a hypothetical RoMMF system. Up to now, most temperature analysis are devoted to capability of uncooled laser diode to keep linearity in a wide temperature range ${ }^{[17,18]}$ allowing competitive prices of the whole system.

The temperature dependence of the 3-dB bandwidth in a 3km-long silica-based MMF link, when the environmental temperature changes, is experimentally investigated and going beyond previous works ${ }^{[19]}$, special attention is given to temperature influence on higher order resonances. About temperature range selection, having in mind environmental variations, in desert areas thermal amplitude between day and night can go up to $35^{\circ} \mathrm{C}$ while in continental climates like in Madrid, it oscillates up to $12^{\circ} \mathrm{C}$. On the hand, annual thermal amplitude is significant; with a record of $87^{\circ} \mathrm{C}$ in extreme continental climate in Mongolia, and in Spain in some regions can go beyond $20^{\circ} \mathrm{C}$ easily. If in-building applications are considered, building regulations and power efficiency policies can imply thermal amplitudes of $18^{\circ} \mathrm{C}$ between occupied and unoccupied periods. And finally, most hubs equipments for wireless applications must fulfilled operation temperature ranges from $0^{\circ} \mathrm{C}$ up to $70^{\circ} \mathrm{C}$, and those temperature range should also applied for MMF under consideration. Having all previous constraints in mind, measurements have been taken from $\mathrm{T}=28^{\circ} \mathrm{C}$ (environment) to $\mathrm{T}=78^{\circ} \mathrm{C}$. The hysteresis cycle of the measurements has also been evaluated. The setup follows the same principle as that of depicted in Fig. 2(a). A FP optical source at $1317 \mathrm{~nm}$ (model SOF-131-C from Accelink) with 2.7nm of linewidth, modulated up to $20 \mathrm{GHz}$ by an external $\mathrm{E} / \mathrm{O}$ modulator has been used to launch optical power into the fiber via a $2 \mathrm{~m}$-long silica-based MMF pacth cord with a mode scrambler thus providing OFL launching condition. The optical power at the 
end of the fiber has been collected by a wide bandwidth InGaAs PIN photodiode (model DSC30S from Discovery Semiconductors), and the frequency response has been analyzed by the LCA. Silica-based specifications follow the same considerations as previously stated in former sections. Test equipment was isolated from the heating source, so that only temperature changes relating to the MMF fiber spool were measured. An average factor of 16 for each temperature test measurement was applied to the following figures. It should be mentioned that all the contributions in literature have been stated that the speckle contrast does not change significantly in Radio-over Multimode Fibre (RoMMF) links with the frequency of the modulating signal, i.e. the Signal-to Noise Ratio, SNR, due to modal noise presents no degradation with the electrical frequency ${ }^{[20]}$. Moreover, the latter work also shows that the speckle contrast is independent from the launching conditions and becomes almost negligible for distances above $3 \mathrm{~km}$, in a $62.5 / 125 \mu \mathrm{m}$ graded-index silica-based MMF optical link, so this effect has not been considered in the following discussion.

Results depicted in Fig. 5 show the normalized frequency response for different temperature cases. Fig. 5(a) illustrates the normalized measured frequency response for a $3 \mathrm{~km}$-long MMF link at $\mathrm{T}=28^{\circ} \mathrm{C}$ and $\mathrm{T}=78^{\circ} \mathrm{C}$ (maximum temperature tested), respectively. The frequency response, in this case, shows slight variations over the spectrum but with power offsets at specific points up to $2 \mathrm{~dB}$, see figure inset. On the other hand, Fig. 5(b) show the hysteresis cycle of the $3 \mathrm{~km}$ long MMF link at $\mathrm{T}=28^{\circ} \mathrm{C}$ (environmental) when heating (forwards) up to $78^{\circ} \mathrm{C}$ and, then, cooling (backwards). From the inset figure the maximum hysteresis deviation is $3-\mathrm{dB}$ at $3 \mathrm{GHz}$, compromising the $3-\mathrm{dB}$ baseband bandwidth in such links. In both cases, an average has been applied, with averaging factor Avg=16.

(a)

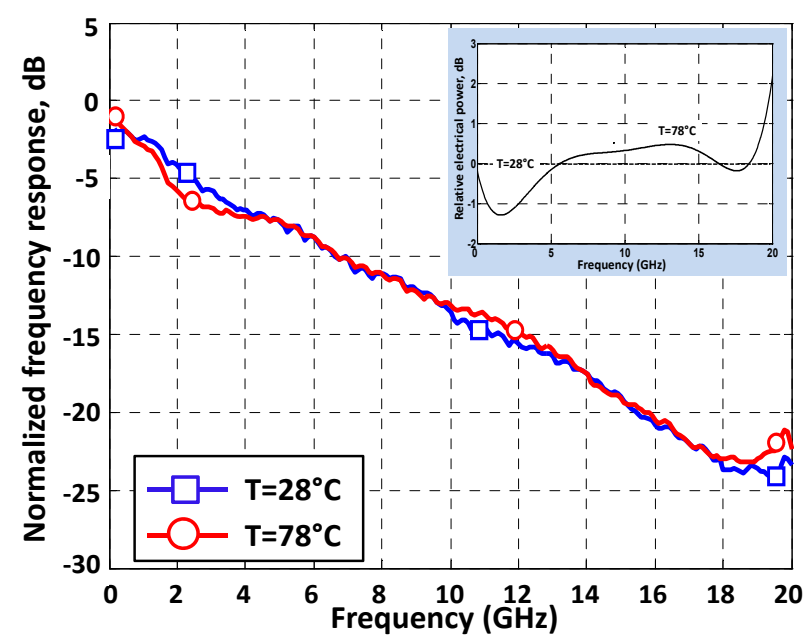

(b)

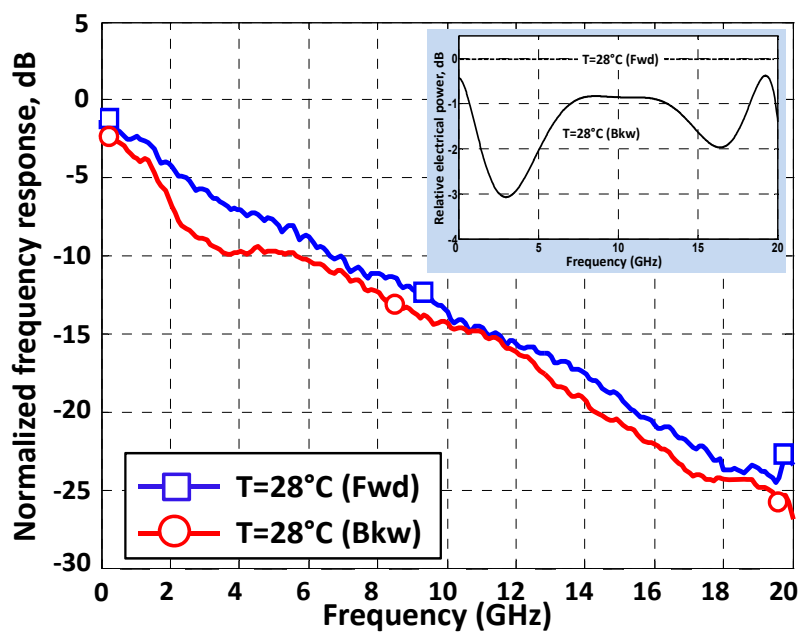

Figure 5. (a) Experimental frequency response of a $3 \mathrm{~km}$-long silica-based $\mathrm{MMF}$ link, at $\mathrm{T}=28^{\circ} \mathrm{C}$ and $\mathrm{T}=78^{\circ} \mathrm{C}, \mathrm{Avg}=16$. ; (b) $\mathrm{Hysteresis}$ of the MMF link at environmental temperature, $\mathrm{Avg}=16$. Fwd: forwards ; Bkw: backwards. Avg: averaging factor. Inset of figures: averaged optical power received, normalized to environmental temperature, $\mathrm{T}=28^{\circ} \mathrm{C}, \mathrm{Avg}=16$.

Considering the above results it is also expected that temperature changes seriously affect the location of the high-order resonances for the implementation of a RoMMF system. Consequently, it would be interesting to evaluate the variation on the performance of the frequency response of an arbitrary single secondary side-lobe with regards to this impairment. For this case, similar tests of frequency response temperature dependence and hysteresis as above have been performed at OFL condition but employing a DFB laser source (model Agilent $81989 \mathrm{~A}$ ) operating at $1550 \mathrm{~nm}$ with $100 \mathrm{kHz}$ of source linewidth, where high-order resonances can be clearly appreciated, see Fig. 3(b). Temperature variations ranged from $\mathrm{T}=22^{\circ} \mathrm{C}$ (environment) to $\mathrm{T}=80^{\circ} \mathrm{C}$. The following set of pictures in Fig. 6 are referred to the first-order resonance, which appears in the frequency response of a 9km-long silica-based MMF link centered around $\left.f_{o}\right|_{1}=3.5 \mathrm{GHz}$ with a 3$\mathrm{dB}$ bandwidth of $2.5 \mathrm{GHz}$ approximately, see Fig. 3(b). This selection allows spectrum allocation in the RoF link of two wireless LAN Access Points (AP) configured for IEEE $802.11 \mathrm{~b}$ operation in the $2.4 \mathrm{GHz}$ band and for IEEE 802.1la operation in the $5.2 \mathrm{GHz}$ band.

From Fig. 6(a) it can be observed that the central frequency of the first-order resonance shows almost no dependence with temperature whereas the $3-\mathrm{dB}$ bandwidth of the resonance at different temperatures suffers from slight deviations. 
The results showed a nominal 3-dB first-resonance bandwidth of $2.5 \pm 0.3 \mathrm{GHz}$, i.e. a deviation from the nominal value of $\pm 12 \%$ in percentage. Conversely, results concerning hysteresis show that high-dependence on temperature cycles of heating (forwards) and cooling (backwards) are noticed. Analyzing the hysteresis, variations of the 3-dB first-resonance bandwidth up to $\pm 0.6 \mathrm{GHz}$ (which means a deviation of $24 \%$ in percentage) were noticed. Furthermore, first-resonance central frequency was displaced even $0.5 \mathrm{GHz}$, which means a deviation of $14 \%$ in percentage from the aforementioned value of $3.5 \mathrm{GHz}$ obtained.

(a)
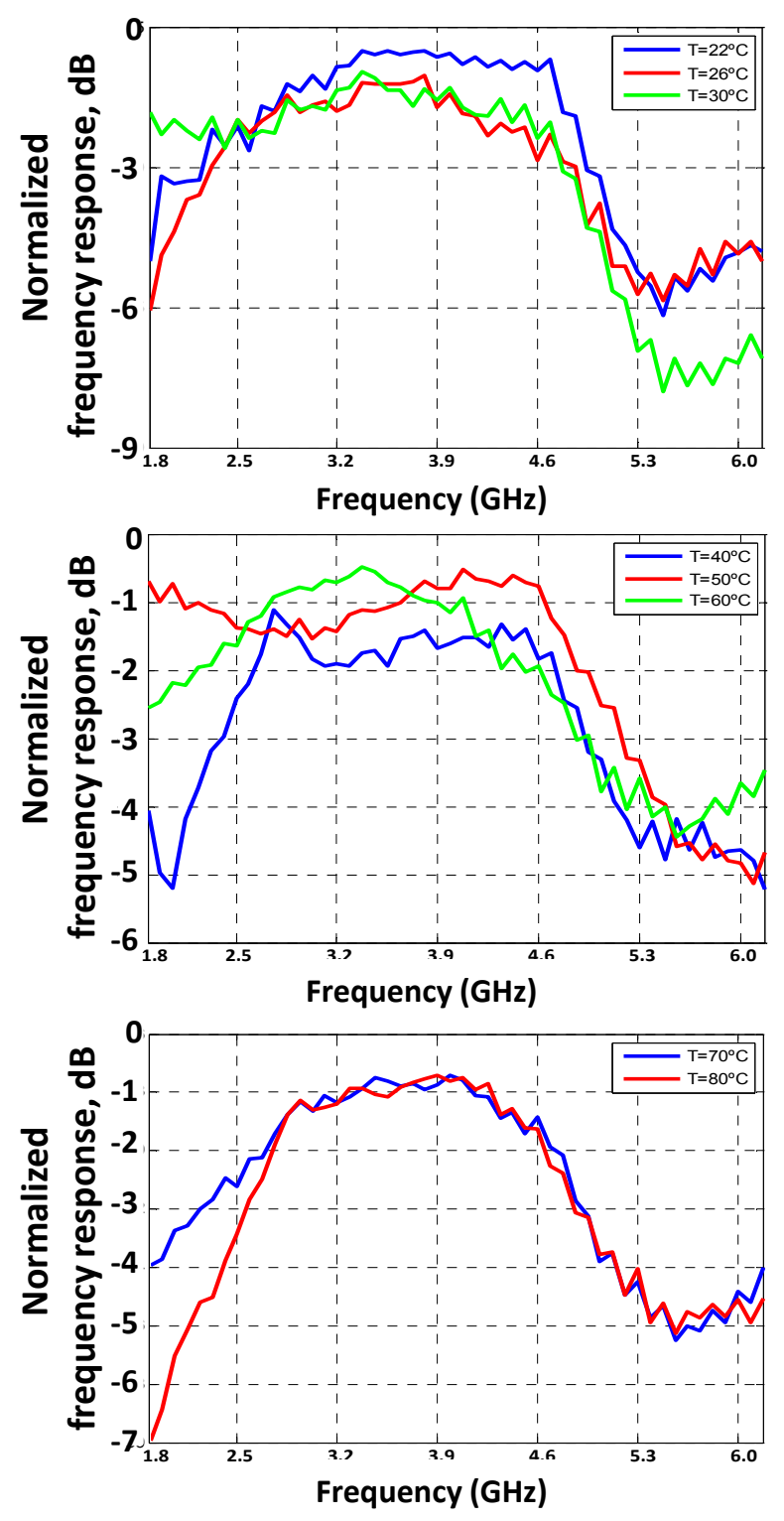

(b)
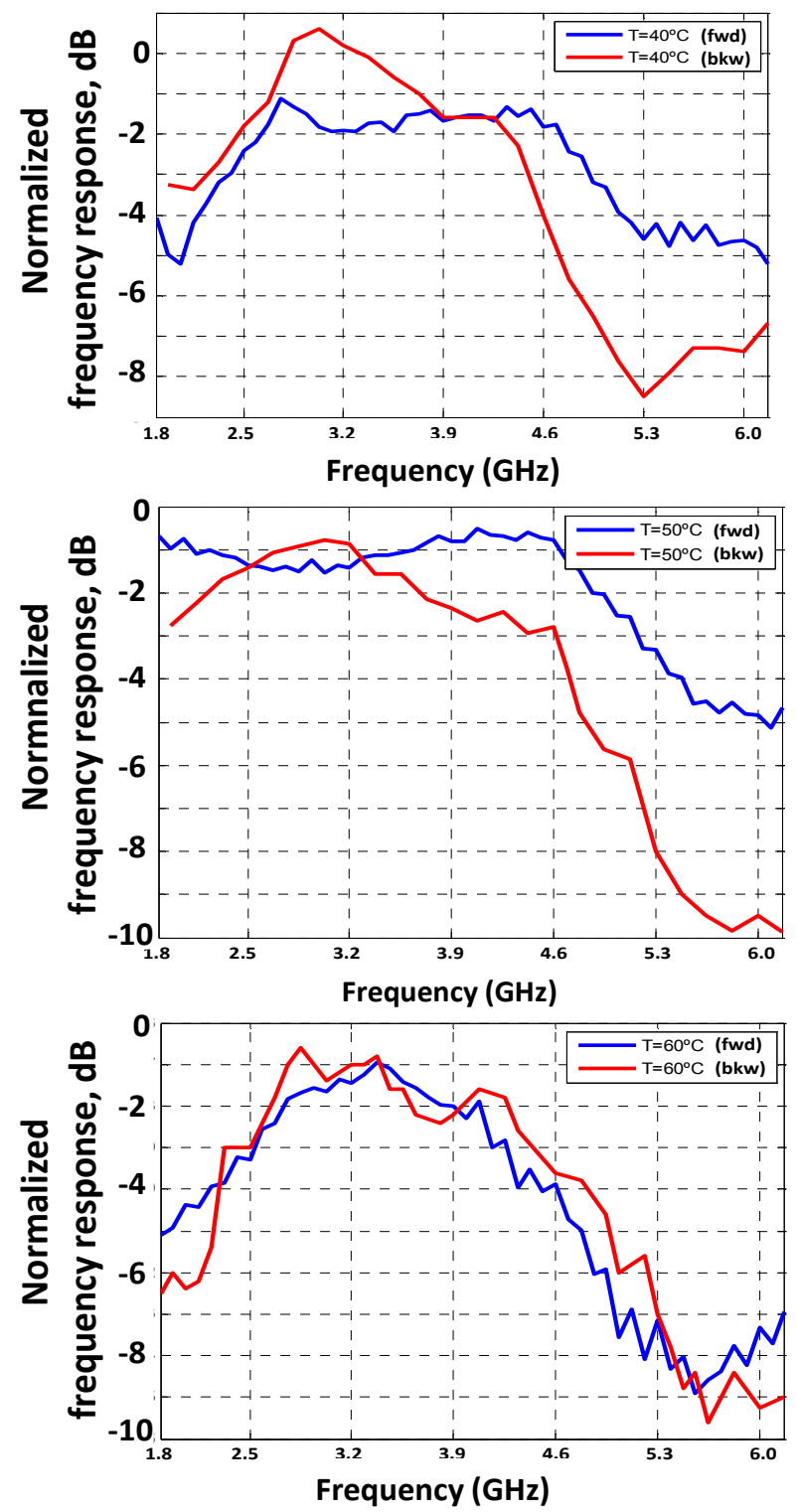

Figure 6. (a) Measured $9 \mathrm{~km}$-long MMF link first-resonance frequency response from $\mathrm{T}=22^{\circ} \mathrm{C}$ to $\mathrm{T}=80^{\circ} \mathrm{C}$. ; (b) Hysteresis of the firstresonance at environmental temperature; fwd: forwards, bkw: backwards.

Table 1 summarizes the first-order resonance measured 3-dB bandwidth $\left(\left.\left.\Delta f_{o}\right|_{1}\right|_{-3 \mathrm{~dB}}\right)$ and central frequency $\left(\left.f_{o}\right|_{1}\right)$ at the different tested temperatures shown in Fig. 6(a) in forwards (fwd) cycle. Both figures show the optical power deviations detected for a single sweep measurement. A statistical analysis of reported measurements show that 3 -dB bandwidth 
versus temperature can be approximated by $\Delta f_{0,1(3 d B)}(T)=1,73012+0,0405611 \mathrm{~T}-0,000453778 T^{2}$ being $\mathrm{T}$ in ${ }^{\circ} \mathrm{C}$ for a range between $20^{\circ} \mathrm{C}$ up to $80^{\circ} \mathrm{C}$, and $3-\mathrm{dB}$ bandwidth in $\mathrm{GHz}$, and a standard deviation of $0,05887 \mathrm{GHz}$.

Table 1. First-order resonance temperature dependence performance (fwd) of the MMF link considered.

\begin{tabular}{c||c|c|c|c|c|c|c||}
\hline \hline \multirow{2}{*}{\begin{tabular}{c} 
Temperature $\left({ }^{\circ} \mathbf{C}\right)$ \\
$\left.f_{o}\right|_{1}(\mathbf{G H z})$ \\
\cline { 2 - 9 } \\
$\left.\left.\Delta f_{o}\right|_{1}\right|_{-3 \mathrm{~dB}}(\mathbf{G H z})$
\end{tabular}} & 3.7 & 3.6 & 3.6 & 3.5 & 3.4 & 3.6 & 3.7 \\
\cline { 2 - 9 } & 2.4 & 2.6 & 2.6 & 2.7 & 2.5 & 2.3 & 2.1 \\
\hline
\end{tabular}

\section{CONCLUSIONS}

To overcome the intrinsic bandwidth limitation and to expand link length capabilities over silica-based MMF fibers, the solution based on the fact that the frequency response in such fiber type does not decrease monotonically outside the baseband is revisited. Moreover, beyond the baseband, there are observed relatively flat frequency ranges suitable for broadband transmission at RF, microwave and millimeter-wave regions as well as passband characteristics in higher frequencies than baseband. Consequently, transmission of multiple-GHz carriers in these MMF links can be featured at certain frequencies albeit a small power penalty, enabling the extension of broadband transmission. This fact has been addressed theoretically and experimentally in this work. However, the potentials of silica-based MMF to support broadband RF, microwave and millimetre-wave transmission over short, intermediate and long distances are yet to be fully known, as its frequency response seems to be unpredictable under arbitrary operating conditions as well as fiber characteristics, and temperature dependence of the MMF frequency response seems to play an important role in Radioover-Multimode Fiber systems.

An experimental analysis of frequency response in silica-based MMF fibers with broadband transmission applications far from baseband exposed to temperature gradients is reported. The silica-based MMF link has been evaluated showing that temperature must be taken into account when selecting a transmission band far from baseband for high-speed data rate applications. Central frequencies of the high-order resonances as well as their respectively 3-dB bandwidths have been experimentally verified to show little dependence (below 10\% deviation) with environmental temperature. However, results concerning hysteresis show that high-dependence on temperature cycles of heating (forwards) and cooling (backwards) are noticed.

To resume, by considering how the temperature affects the silica-based MMF link it is possible to avoid the negative influence of this parameter over the high-order resonances and, consequently, to maximize performance of RoMMF broadband transmissions by means of an accurate addressing of the high-order secondary lobes in the MMF frequency response. However, in real situations where an optical link is deployed it would be far more useful having predictive accurate models that consider the environmental temperature changes; so fault link prevention can be developed.

\section{REFERENCES}

[1] Toma, T., Takizuka, H., Kanou, M., Taniguchi, T. and Koike Y., "Dual full high definition 3D video real-time communication system," Proc. International Conference on Plastic Optical Fibers (ICPOF), 475-479 (2011).

[2] Parker, M. C., Walker, S. D., Llorente, R., Morant, M., Beltrán, M., Möllers, I., Jäger, D., Vázquez, C., Montero, D. S., Librán, I., Mikroulis, S., Karabetsos, S. and Bogris A., "Radio-over-fibre technologies arising from the building the future optical network in Europe (BONE) project," IET Optoelectronics 4(6), 247-259 (2010).

[3] W. Alliance, "Worldwide regulatory status [Online]. Available: http://www.wimedia.org."

[4] Koonen, A. M. J. and Garcia Larrodé, M., "Radio-Over-MMF Techniques;Part II: Microwave to MillimeterWave Systems," J. Lightwave Technol. 26(15), 2396-2408 (2008). 
[5] Gasulla, I. and Capmany, J., "Transfer function of multimode fiber links using an electric field propagation model: Application to Radio over Fibre Systems," Opt. Express 14(20), 9051-9070 (2006).

[6] Montero, D. S. and Vázquez, C., "Analysis of the electric field propagation method: theoretical model applied to Perfluorinated Graded-Index Polymer Optical Fiber links," Optics Letters 36(20), 4116-4118 (2011).

[7] Ingham, J. D., Webster, M., Wake, D., Seeds, A. J., Penty, R. V. and White, I. H., "Bidirectional Transmission of 32-QAM Radio Over a Single Multimode Fibre Using 850-nm Vertical-Cavity Half-Duplex Transceivers," Proc. 28th European Conference on Optical Communication (ECOC 2002), 1-2 (2002).

[8] Gasulla, I. and Capmany, J., "Simultaneous baseband and radio over fiber signal transmission over a $5 \mathrm{~km}$ MMF link," International Topics Meeting on Microwave Photonics, 209-212 (2008).

[9] García Larrodé, M., Koonen, A. M. J. and Vegas Olmos, J. J., "Overcoming Modal Bandwidth Limitation in Radio-over-Multimode Fiber Links," IEEE Photon. Technol. Lett. 18, 2428-2430 (2006).

[10] Saleh, B. E. A. and Abdula, R. M., "Optical Interference and Pulse Propagation in Multimode Fibers," Fiber Integr. Opt. 5, 161-201 (1985).

[11] Yabre, G., "Theoretical investigation on the dispersion of graded-index polymer optical fibers," J. Lightwave Technol. 18(6), 869-877 (2000).

[12] Hartmann, P., Xin, Q., Wonfor, A., Penty, R. V. and White, I. H., "1-20 GHz Directly Modulated Radio over MMF Link," International Topics Meeting on Microwave Photonics, 95-98 (2005).

[13] Khawaja, B. A. and Cryan, M. J., "Characterisation of Multimode Fibres for Use in Millimetre Wave RadioOver-Fibre Systems," Asia-Pacific Microwave Conference, 1-4 (2007).

[14] Gasulla, I. and Capmany, J., "Transmission of high-frequency radio over fibre signals through short and middle reach Multimode Fibre links using a low-linewidth laser," International Topics Meeting on Microwave Photonics, 116-119 (2007).

[15] Hamza, A. A., Sokkar, T. Z. N., El-Farahaty, K. A. and El-Dessouky H. M., "Influence of temperature on the optical and structural properties along the diameter of optical fibers," Opt. and Lasers Eng. 41, 261-275 (2004).

[16] Tarrach, F., Ch'hayder, A., and Guermazi, S., "Influence of thermal aging on optical fiber properties," Opt. Eng. 47, 0650061-4 (2008).

[17] Ingham, J., Webster, M., Wonfor, A., Penty, R. V., White, I. H. and White, K., "Wide-frequency-range operation of a high-linearity uncooled DFB laser for next-generation radio-over-fiber," Optical Fiber Communications Conference (OFC) 752, 754-756 (2003).

[18] Seeds A. J. and Ismail, T., "Broadband Access Using Wireless Over Multimode Fiber Systems," J. Lightwave Technol. 28(16), 2430-2435 (2010).

[19] Montero, D. S., Gasulla, I., Möllers, I., Jäger, D., Capmany, J. and Vázquez, C., "Experimental analysis of temperature dependence in multimode optical fiber links for radio-over-fiber applications," International Conference on Transparent Optical Networks (ICTON), 1-4 (2009).

[20] Gasulla, I. and Capmany, J., "Modal noise impact in Radio over Fiber multimode fiber links," Opt. Express 16, 121-126 (2008). 